\title{
Terahertz Communication: The Opportunities of Wireless Technology Beyond 5G
}

\author{
Hadeel Elayan ${ }^{1}$, Osama Amin ${ }^{2}$, Raed M. Shubair ${ }^{3}$, and Mohamed-Slim Alouini ${ }^{2}$ \\ ${ }^{1}$ Department of Electrical and Computer Engineering Khalifa University, UAE \\ ${ }^{2}$ Computer, Electrical and Mathematical Sciences and Engineering (CEMSE) Division, \\ King Abdullah University of Science and Technology (KAUST), KSA \\ ${ }^{3}$ Research Laboratory of Electronics, Massachusetts Institute of Technology, USA
}

\begin{abstract}
Over the past years, carrier frequencies used for wireless communications have been increasing to meet bandwidth requirements. The engineering community witnessed the development of wide radio bands such as the millimeter-wave ( $\mathrm{mmW}$ ) frequencies to fulfill the explosive growth of mobile data demand and pave the way towards $5 \mathrm{G}$ networks. Other research interests have been steered towards optical wireless communication to allow higher data rates, improve physical security and avoid electromagnetic interference. Nevertheless, a paradigm change in the electromagnetic wireless world has been witnessed with the exploitation of the Terahertz ( $\mathrm{THz})$ frequency band $(0.1-10 \mathrm{THz})$. With the dawn of $\mathrm{THz}$ technology, which fills the gap between radio and optical frequency ranges, ultimate promise is expected for the next generation of wireless networks. In this paper, the light is shed on a number of opportunities associated with the deployment of the $\mathrm{THz}$ wireless links. These opportunities offer a plethora of applications to meet the future communication requirements and satisfy the ever increasing user demand of higher data rates.

Index Terms-Terahertz communication, wireless technology, next-generation wireless networks, beyond 5G
\end{abstract}

\section{INTRODUCTION}

Wireless data traffic has been witnessing unprecedented expansion in the past few years. In particular, mobile data traffic is expected to increase sevenfold between 2016 and 2021, whereas video traffic is anticipated to experience a threefold increase during the same time period [1]. Such remarkable growth of wireless traffic has driven the urge to investigate suitable regions in the radio spectrum to fulfill users' accelerating demands. To this end, the Terahertz (THz) frequency band (0.1-10 THz) started to receive considerable attention in the research fraternity. Big data wireless cloud, ultra-fast wireless download, and seamless data transfer are all evolutions that will transform the telecommunications landscape and revolutionize the way people communicate and access information.

The $\mathrm{THz}$ frequency band promises extensive bandwidth, which theoretically reaches up to several $\mathrm{THz}$, resulting in a potential capacity in the order of Terabits per second [2]. Hence, the bandwidth supplied is one order of magnitude above millimeter-wave ( $\mathrm{mmW}$ ) systems. $\mathrm{THz}$ signals also allow higher link directionality and offer lower eavesdropping chances when compared to their millimeter counterparts [3].
Analysis of the $\mathrm{THz}$ band indicates that these frequencies also possess a set of advantages in comparison to optical frequencies. $\mathrm{THz}$ waves are considered candidates for uplink communication. They permit non-line-of-sight (NLoS) propagation [4] and act as good substitutes under inconvenient climate conditions including fog, dust and turbulence [5]. In addition, the $\mathrm{THz}$ frequency band is not impacted by ambient noise arising from optical sources nor is it associated with any health restrictions or safety limits [6]. Table I provides a comparison between the $\mathrm{THz}$ frequency band and other existing technologies to showcase the ultimate promise this band holds towards the next generation of wireless networks.

In 2008, the IEEE 802.15 established the "THz Interest Group" as a milestone towards the standardization of $\mathrm{THz}$ communications operating in frequency bands between 275 and $3000 \mathrm{GHz}$. A group named "the task group 3d (TG3d)" has been formed in 2014 to modify the 802.15.3 metrics in an aim to target 100 Gigabits per second for switched pointto-point links. A plethora of applications fall under this category including wireless data centers, backhauling/fronthauling as well as close-proximity communication such as kiosk downloading and device-to-device (D2D) communication. The allocation of frequencies beyond $275 \mathrm{GHz}$ will actually be an item in the agenda of the "2019 World Radiocommunication Conference" under the International Telecommunication Union [7].

As a result of the exponential growth of wireless traffic, the demand for higher bandwidth will continue to increase and never seem to subside before the capacity of the generation beyond $5 \mathrm{G}$ technology has reached its upper limit. In this paper, we highlight a number of opportunities correlated with the deployment of the $\mathrm{THz}$ frequency band. These opportunities are demonstrated as applications that will facilitate a refined wireless experience. Thus, the foundation for a resilient communication infrastructure will eventually be established to cope with users' demands. The rest of the paper is organized as follows. In Section II, the system architecture of $\mathrm{THz}$ wireless communication is presented in which we review the $\mathrm{THz}$ transceivers and channel models available in the literature. In Section III, a number of opportunities associated with the deployment of the $\mathrm{THz}$ frequency band are demonstrated in an aim to effectively meet the needs of future networks. Finally, we conclude the paper in Section IV.(5) 
TABLE I

COMPARISON BETWEEN WiRELESS COMMUNiCATION TECHNOLOGIES

\begin{tabular}{|l|l|l|l|l|l|}
\hline Technology & $\mathbf{m m W}$ & THz Band & Infrared & Visible Light Communication (VLC) & Ultra-Violet \\
\hline Frequency Range & $30 \mathrm{GHz}-300 \mathrm{GHz}$ & $100 \mathrm{GHz}-10 \mathrm{THz}$ & $10 \mathrm{THz}-430 \mathrm{THz}$ & $430 \mathrm{THz}-790 \mathrm{THz}$ & $790 \mathrm{THz}-30 \mathrm{PHz}$ \\
\hline Range & Short range & Short/Medium range & Short/Long range & Short range & Short range \\
\hline Power Consumption & Medium & Medium & Relatively low & Relatively low & Expected to be low \\
\hline Network Topology & Point to Multi-point & Point to Multi-point & Point to Point & Point to Point & Point to Multi-point \\
\hline Noise Source & Thermal noise & Thermal noise & Sun/Ambient Light & Sun/Ambient Light & Sun/Ambient Light \\
\hline Weather Conditions & Robust & Robust & Sensitive & - & High \\
\hline Security & Medium & High & High & To be determined \\
\hline
\end{tabular}

\section{System ARChitecture OF THz COMMUNiCATIONS}

To realize an operational $\mathrm{THz}$ communication system, advancements from both the devices and channel modeling perspectives are required. This will eventually result in unprecedented progress in the $\mathrm{THz}$ wireless field and will lead to extensive growth in $\mathrm{THz}$ applications.

\section{A. Terahertz Transceivers}

$\mathrm{THz}$ waves are considered one of the least investigated and rarely capitalized regions in the electromagnetic spectrum. The fact that these waves are located between the $\mathrm{mmW}$ and optical frequency bands allows both electronics-based and photonicsbased technologies to be deployed for their generation. As for electronic devices, recent progress in nano-fabrication technologies facilitated the progress of semiconductor devices that function in the $\mathrm{THz}$ frequency band. Such devices include Gallium Arsenide and Indium Phosphide electronics [8] as well as various Silicon-based technologies [9]. Recently, the authors in [10], presented an innovative Silicon architecture, which permits its scalability, to allow signal synthesis and enable the shaping of $\mathrm{THz}$ waves in a single microchip. Such contribution is fundamental as much of the challenge on the THz-generation end lies in creating a wide range of wavelengths within the $\mathrm{THz}$ band, particularly in a microchip. In terms of photonics-devices, the feeding mechanism of the optical fibers to the $\mathrm{THz}$ emission circuits is a key factor in achieving higher data rates. In specific, photonics-based techniques offer a unique opportunity to attract early users or customers by demonstrating the technology through lasers, modulators and photodiodes which are all already available in the market. These devices also permit the ability to deal with multi-carrier $\mathrm{THz}$ channels due to the carrier switching capabilities that facilitate the radio/optical interface in hybrid networks [11]. Table II provides a brief comparison between the current $\mathrm{THz}$ technologies available in the literature. Apart from the source, the antenna needs due concentration. A large array of antenna elements can be arranged on terminal devices as $\mathrm{THz}$ frequencies allow small antenna size. Therefore, diversity gain as well as antenna directivity gain over Multiple-Input-Multiple-Output (MIMO) approaches are achieved. Actually, the concept of Ultra-Massive MIMO by
$1024 \times 1024$ has been introduced as an approach to increase communication distance in $\mathrm{THz}$ systems [12].

In addition, most of the conventional materials used at lower frequencies, namely the microwave and $\mathrm{mmW}$ ranges, cannot efficiently respond to high frequencies and typically exhibit large losses at THz. Hence, an alternative to $\mathrm{THz}$ band compact transceivers relies on using graphene [13]. Practical applications also require electromagnetically reconfigurable materials which constitute the basis of all active $\mathrm{THz}$ components. In this context, graphene has received the recognition of the scientific community as a result of its strengths in atomic thickness, tunability and high kinetic inductance where it is possible to reform $\mathrm{THz}$ electromagnetic waves by using thin graphene layers [14]. Consequently, graphenebased $\mathrm{THz}$ components have shown very promising results in terms of generating, modulating as well as detecting $\mathrm{THz}$ waves [15]. In specific, graphene plasmonics open the door towards optoelectronic applications in the $\mathrm{THz}$ frequency range [16]. This results in having novel devices with unrivaled functioning capabilities, such as subwavelength guiding structures, nanoantennas, superlenses, hyperlenses, and light concentrators.

\section{B. Terahertz Channel Model}

In order to be capable of understanding the peculiarities of any wireless channel before deployment, it is necessary to adopt a specific model that perfectly describes the behavior of the channel. At the $\mathrm{THz}$ frequency band, the channel suffers from high atmospheric absorption, which results from water vapor molecules. In addition to atmospheric attenuation, free-space path loss is considered physically unavoidable. Consequently, the $\mathrm{THz}$ band channel is considered highly frequency selective [20]. Therefore, the transmission distance is limited by attenuation and the appropriate carrier frequency is determined according to the application. Fig. 1 indicates the atmospheric attenuation levels impacting the propagating $\mathrm{THz}$ waves. However, a closer look indicates that despite the existence of absorption peaks centered at specific frequencies, the availability of transmission windows allows establishing viable communication at the $\mathrm{THz}$ frequency band.

The current literature on channel modeling in the $\mathrm{THz}$ band has been limited and restricted to indoor environments as we 
TABLE II

Summary of Terahertz Band Technologies (100 GHz - 10 THz) Presented in the Literature

\begin{tabular}{|l|l|l|c|}
\hline Technology & Carrier Frequency & Data Rate & Reference \\
\hline Si-electronics & Up to $240 \mathrm{GHz}$ & $10 \mathrm{Gbit} / \mathrm{s}$ & {$[9]$} \\
\hline GaAs/InP electronics & Up to $300 \mathrm{GHz}$ & $64 \mathrm{Gbit} / \mathrm{s}$ & {$[17]$} \\
\hline Photonics-based (Single Channel) & $300-500 \mathrm{GHz}$ & $160 \mathrm{Gbit} / \mathrm{s}$ & {$[18]$} \\
\hline Photonics-based (Multi-band Channel) & $300-500 \mathrm{GHz}$ & $>160 \mathrm{Gbit} / \mathrm{s}$ & {$[19]$} \\
\hline
\end{tabular}

still lack models that emulate outdoor environments. Basically, most models available in the literature can be categorized into either path loss or ray-tracing models. Examples of these works include [20]-[22]. The first statistical model for THz channels, spanning the range between 275 and $325 \mathrm{GHz}$, has been provided in [23]. The given model relies on performing extensive ray-tracing simulations to obtain the channel statistical parameters. However, the drawback of this model is that the information regarding the channel statistics such as the correlation function and power-delay profile cannot be perceived easily. To address these issues, the authors in [24], [25] presented a geometrical statistical model for D2D scatter channels at the sub-THz band. These models mimic the scattering and reflection patterns in a sub-THz D2D environment. In addition, unlike traditional channel measurements, scenariospecific measurements are also available in the literature. The authors in [26] presented a stochastic model for kiosk applications in the $\mathrm{THz}$ band, specifically between 220 and $340 \mathrm{GHz}$. Channel measurements have been conducted using a 3D ray-tracing simulator to extract channel characteristics of three different kiosk application scenarios. Designing closeproximity communication systems at $\mathrm{THz}$ frequencies will actually be permitted through the proposed channel model. Another recent study on the statistical channel characterization of a THz scenario has been presented in [27]. The study tackles the frequency range between 240 and $300 \mathrm{GHz}$; yet, it is one of the first to provide single-sweep $\mathrm{THz}$ measurement results. The measured data allows finer temporal details to be obtained aiding the design of reliable transceiver systems including antenna misalignment problems.

\section{OPPORTUNITIES OF TERAHERTZ WiRELESS COMMUNICATION}

In this section, we focus on a number of foreseen opportunities that illustrate the future of $\mathrm{THz}$ wireless and the thrust in exploiting a spectral band which was once referred to as a "gap".

\section{A. Hybrid Terahertz-Optical Wireless Communication Links}

The concept of a hybrid radio frequency/free space optical (FSO) system has attracted a lot of research recently [29]. In this context, a THz/optical link is expected to be a viable approach for future wireless communication. Two hybrid schemes exist for such setup, namely, parallel and serial. For the parallel hybrid scenario, two communication links are present and may be used in either one-way or twoway fashion. The backhaul link is an example of a oneway hybrid scenario where either FSO or $\mathrm{THz}$ technology is

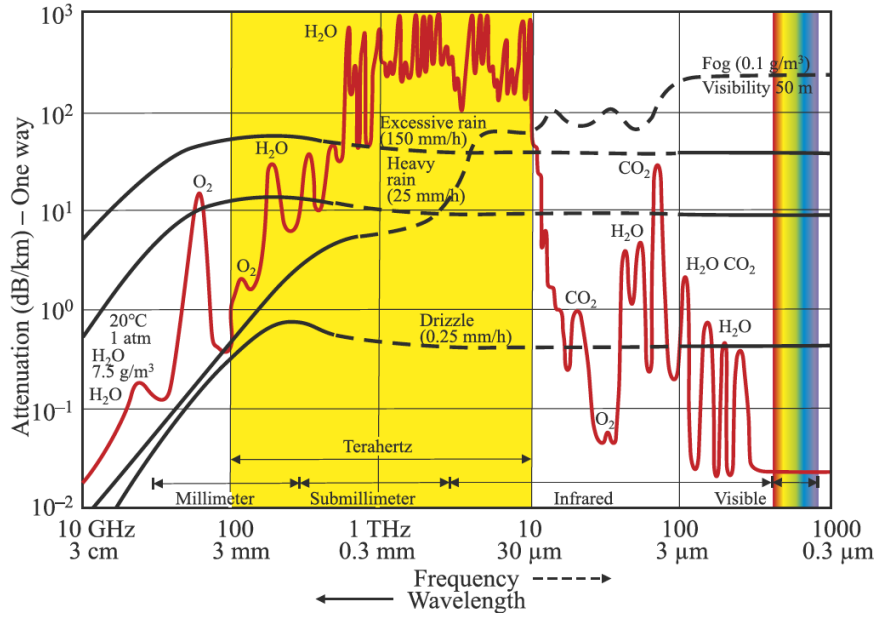

Fig. 1. Comparison between the attenuation impact of different environmental effects on different technologies [28].

selected based on the weather conditions. For instance, the FSO technology is chosen when the weather is clear with negligible wind to guarantee robust beam-tracking. On the other hand, $\mathrm{THz}$ comes to the scene in foggy and/or windy weather conditions, where a reliable link can be established under these conditions. As for the two-way communication setup, $\mathrm{THz}$ can play reliable performance in the uplink of VLC cells without necessitating the position, acquisition and tracking (PAT) techniques associated with the usage of the infrared uplink service. For the serial communication scenario, multi-hop communication links are used, in which each link is optimized to operate using the technology which maximizes the system performance.

\section{B. Terahertz Automotive Applications}

In recent years, unmanned autonomous vehicles (UAVs) have become accessible to the public resulting in numerous applications in both civilian and commercial domains. Examples include weather control, forest fire recognition, traffic detection, cargo transport, emergency search and rescue as well as communication broadcasting [30]. To achieve such applications, UAVs ought to have a reliable communication link available at all times. Interestingly, the impact of moisture is imperceptible for heights above $16 \mathrm{~km}$; thus, $\mathrm{THz}$ attenuation is trivial. As such, $\mathrm{THz}$ can become a strong candidate to establish reliable communications for different UAV application scenarios.

Compared to $\mathrm{FSO}$, the $\mathrm{THz}$ frequency band is considered a suitable technology since it does not only achieves high- 
capacity UAV-UAV wireless backhaul but also provides a better alternative in mitigating the high mobility environment of UAVs. In fact, as a result of mobility, communication links which suffer from the Doppler effect are minimized as the carrier frequency increases. Therefore, the $\mathrm{THz}$ frequency band can allow high-speed communication links among two dynamic locations. In addition, UAVs require short-distance secure links to obtain commands or transmit data before scattering to pursue either their remote controlled or self-governing missions. Thus, $\mathrm{THz}$ links are considered a reliable venue for interchanging safety-critical data amid UAVs as well as among the UAV and the ground control stations. Actually, the massive bandwidth of $\mathrm{THz}$ systems enables distinct protection actions against different standoff attacks such as jamming and provides the ability to completely hide information exchange. Furthermore, $\mathrm{THz}$ links could be also utilized between UAVs and airplanes in order to support internet for flights instead of using the satellite service. In this way, the UAV will act as a switchboard in the sky serving as an intermediary between the ground station and the airplane.

\section{Terahertz for Enhanced Data Centers Performance}

Consumers increasing demand for cloud applications stimulated competition among data centers to provide users with an enhanced performance experience. This is achieved by accommodating a large number of servers as well as supplying sufficient bandwidths for a wide array of applications. Yet, it is quite challenging to handle traffic bursts as a result of static links and finite network interfaces by relying solely on fixed wired networks. Nevertheless, wireless networking, which serves as a correlative technology to Ethernet, has the adaptability and efficiency required to contribute possible means to tackle the problem [31]. It is to be noted however that wireless transmissions may suffer from short distances and intolerance to blockage resulting in the depletion of the capability of data centers if all wires are replaced. This is why augmenting the data center network with wireless flyways instead of substituting all cables is a better alternative [32]. The authors in [33] suggested using $\mathrm{THz}$ links in data centers as a parallel technology. Due to such deployment, performance improvements in data centers coupled with massive savings in cable prices can be attained without compromising any throughput. In specific, atmospheric data has been utilized to model the $\mathrm{THz}$ channel, where the authors recommended using a bandwidth of $120 \mathrm{GHz}$ for data centers applications. It is to be emphasized that in such application, $\mathrm{THz}$ links outperforms both $\mathrm{mmW}$ and infrared technologies since the former has limited bandwidth while the latter suffers from huge complexity in coherent detection as well as limitations in its square law detectors.

\section{Terahertz Communications for Mobile HetNets}

Although the $\mathrm{THz}$ frequency band suffers from high path loss and requires highly directional antennas, these drawbacks could turn into favorable attributes when used in the femtocell regime. The deployment of femtocells decreases the distance among both the active base-station and the user, while maintaining high SINR at the receiver. THz femtocell base-stations not only improve the principle of frequency reuse but also increase the capacity of the $\mathrm{THz}$ band systems. Thus, these access points, also referred to as Low-Power Nodes (LPN), are utilized as portals to in-home service and automation, metro-stations, shopping malls, traffic lights and many other applications. Such potential opens the door towards a new era of $\mathrm{THz}$ communications for mobile heterogeneous networks (HetNets). Depending on the environment, the quality and type of communication service, picocells and femtocells will be collocated within the macrocell footprint.

To enhance the $\mathrm{THz}$ femtocell experience, machine learning (ML) could be used as a supporting artificial intelligence tool to improve the functionality of smart radio terminals. Actually, ML can be widely used in modeling a number of technical problems including large scale MIMOs, D2D networks as well as HetNets [34]. In particular, as $\mathrm{THz}$ communication consists of access points in either ubiquitous WiFi networks or base-station clustering, unsupervised learning strategies are required. In addition, reinforcement learning is also of significant importance in $\mathrm{THz}$ communication as the self-organization capability is needed to enable femtocells to autonomously identify the available spectrum and tune their parameters accordingly. Such cells will therefore function under the limits of evading interference and satisfying QoS demands [35].

\section{E. Terahertz 3D Beamforming Technology}

3D MIMO technology seems to be the future of $\mathrm{THz}$ wireless. Indeed, the real-world channel features 3D characteristics, rendering 2D MIMO techniques suboptimum [36]. To mitigate the inevitable path loss experienced by the THz channel, 3D beamforming appears as a solution to enable the construction of directional beams, prolong the communication range and reduce interference. Hence, the vertical beam pattern possesses a complete active correspondence per resource and per user equipment. 3D beamforming can also increase the strength of the signal by allowing the vertical main lobe to be located precisely at the receiver at any position. By adopting beam coordination or MIMO schemes, the alteration in vertical dimension has the potential to capitalize on additional diversity or spatial separation. This will eventually result in either boosting the quality of the signal or increasing the number of users supported concurrently [37].

\section{CONCLUSION}

A historic breakthrough for science and technology is anticipated through the utilization of $\mathrm{THz}$ wireless communication. The $\mathrm{THz}$ frequency band $(0.1-10 \mathrm{THz})$ provides a vast array of applications to meet the communication demands and market requirements beyond $5 \mathrm{G}$ networks. In this paper, a number of foreseen opportunities associated with the deployment of the $\mathrm{THz}$ frequency band are demonstrated. These include: hybrid THz/optical wireless link, $\mathrm{THz}$ automotive applications, $\mathrm{THz}$ for enhanced data centers performance, $\mathrm{THz}$ communications for mobile HetNets, as well as $\mathrm{THz} 3 \mathrm{D}$ beamforming technology. One factor in the drive towards $\mathrm{THz}$ wireless technology 
that should be given further emphasis is the growing trend of Software Defined Radio and Software Defined Networking. These architectures signify that the process of upgrading the existing 4G/5G technologies to networks beyond $5 \mathrm{G}$ will be easier with cloud-based resources and software load enabling equipments. Another important point that should always be highlighted is the fact that $\mathrm{THz}$ research is built on many areas of science; therefore, the coordination and integration between various disciplines will pave the road towards ultra-high speed communications.

\section{REFERENCES}

[1] C. V. N. Index, "Cisco visual networking index: Forecast and methodology 2015-2020," White paper, CISCO, 2015.

[2] I. F. Akyildiz, J. M. Jornet, and C. Han, "TeraNets: ultra-broadband communication networks in the terahertz band," IEEE Commun. Mag., vol. 21, no. 4, pp. 130-135, Aug. 2014.

[3] J. Federici and L. Moeller, "Review of terahertz and subterahertz wireless communications," J. Appl. Phys., vol. 107, no. 11, p. 6, 2010.

[4] A. Moldovan, M. A. Ruder, I. F. Akyildiz, and W. H. Gerstacker, "Los and nlos channel modeling for terahertz wireless communication with scattered rays," in 2014 IEEE GC Wkshps, Dec. 2014, pp. 388-392.

[5] K. Su, L. Moeller, R. B. Barat, and J. F. Federici, "Experimental comparison of terahertz and infrared data signal attenuation in dust clouds," J. Opt. Soc. Am. A, vol. 29, no. 11, pp. 2360-2366, Nov. 2012. [Online]. Available: http://josaa.osa.org/abstract.cfm?URI=josaa29-11-2360

[6] P. H. Siegel, "Terahertz technology in biology and medicine," IEEE Trans. Microw. Theory Tech., vol. 52, no. 10, pp. 2438-2447, Oct. 2004.

[7] "Ieee standard for high data rate wireless multi-media networksamendment 2: $100 \mathrm{gb} / \mathrm{s}$ wireless switched point-to-point physical layer," IEEE Std 802.15.3d-2017 (Amendment to IEEE Std 802.15.3-2016 as amended by IEEE Std 802.15.3e-2017), pp. 1-55, Oct. 2017.

[8] T. Nagatsuma, "Terahertz technologies: present and future," IEICE ELEX, vol. 8, no. 14, pp. 1127-1142, 2011.

[9] M. Fujishima, S. Amakawa, K. Takano, K. Katayama, and T. Yoshida, "Tehrahertz cmos design for low-power and high-speed wireless communication," IEICE Trans. Electron., vol. 98, no. 12, pp. 1091-1104, 2015.

[10] X. Wu and K. Sengupta, "Dynamic waveform shaping with picosecond time widths," IEEE J. Solid-State Circuits, vol. 52, no. 2, pp. 389-405, Feb 2017.

[11] T. Nagatsuma, G. Ducournau, and C. C. Renaud, "Advances in terahertz communications accelerated by photonics," Nat. Photonics, vol. 10, no. 6, pp. 371-379, 2016.

[12] I. F. Akyildiz and J. M. Jornet, "Realizing ultra-massive mimo (1024× 1024) communication in the (0.06-10) terahertz band," Nano Comm. Net., vol. 8, pp. 46-54, 2016

[13] J. M. Jornet and I. F. Akyildiz, "Graphene-based plasmonic nanotransceiver for terahertz band communication," in 2014 IEEE EuCAP Apr. 2014, pp. 492-496.

[14] C. J. Docherty and M. B. Johnston, "Terahertz properties of graphene," J Infrared Millim THz Waves, vol. 33, no. 8, pp. 797-815, 2012.

[15] M. Hasan, S. Arezoomandan, H. Condori, and B. Sensale-Rodriguez, "Graphene terahertz devices for communications applications," Nano Comm. Net., vol. 10, pp. 68-78, 2016.

[16] L. Ju, B. Geng, J. Horng, C. Girit, M. Martin, Z. Hao, H. A. Bechtel, X. Liang, A. Zettl, Y. R. Shen et al., "Graphene plasmonics for tunable terahertz metamaterials," Nat. Nanotechnol., vol. 6, no. 10, p. 630, 2011.

[17] I. Kallfass, I. Dan, S. Rey, P. Harati, J. Antes, A. Tessmann, S. Wagner, M. Kuri, R. Weber, H. Massler et al., "Towards mmic-based 300ghz indoor wireless communication systems," IEICE Trans. Electron., vol. 98, no. 12, pp. 1081-1090, 2015.

[18] X. Yu, S. Jia, H. Hu, M. Galili, T. Morioka, P. U. Jepsen, and L. K. Oxenløwe, "160 gbit/s photonics wireless transmission in the 300-500 ghz band," APL Photon., vol. 1, no. 8, p. 081301, 2016.

[19] X. Pang, S. Jia, O. Ozolins, X. Yu, H. Hu, L. Marcon, P. Guan, F. D. Ros, S. Popov, G. Jacobsen, M. Galili, T. Morioka, D. Zibar, and L. K. Oxenkwe, "260 gbit/s photonic-wireless link in the thz band," in 2016 IEEE IPC, Oct. 2016, pp. 1-2.

[20] J. M. Jornet and I. F. Akyildiz, "Channel modeling and capacity analysis for electromagnetic wireless nanonetworks in the terahertz band," IEEE Trans. Commun., vol. 10, no. 10, pp. 3211-3221, Oct. 2011.
[21] B. Peng, S. Rey, and T. Kürner, "Channel characteristics study for future indoor millimeter and submillimeter wireless communications," in 2016 IEEE EuCAP, Apr. 2016, pp. 1-5.

[22] C. Han, A. O. Bicen, and I. F. Akyildiz, "Multi-ray channel modeling and wideband characterization for wireless communications in the terahertz band," IEEE Trans. Commun., vol. 14, no. 5, pp. 2402-2412, May 2015.

[23] S. Priebe and T. Kurner, "Stochastic modeling of thz indoor radio channels," IEEE Trans. Commun., vol. 12, no. 9, pp. 4445-4455, Sep. 2013.

[24] S. Kim and A. Zajić, "Statistical modeling of thz scatter channels," in 2015 IEEE EuCAP, May 2015, pp. 1-5.

[25] _ "Statistical modeling and simulation of short-range device-todevice communication channels at sub-thz frequencies," IEEE Trans. Commun., vol. 15, no. 9, pp. 6423-6433, Sep. 2016.

[26] D. He, K. Guan, A. Fricke, B. Ai, R. He, Z. Zhong, A. Kasamatsu, I. Hosako, and T. Kürner, "Stochastic channel modeling for kiosk applications in the terahertz band," IEEE Trans. THz Sci. and Technol., 2017.

[27] A. R. Ekti, A. Boyaci, A. Alparslan, . Ünal, S. Yarkan, A. Görçin, H. Arslan, and M. Uysal, "Statistical modeling of propagation channels for terahertz band," in 2017 IEEE CSCN, Sep. 2017, pp. 275-280.

[28] A. Rogalski and F. Sizov, "Terahertz detectors and focal plane arrays," Opto-Electron. Rev., vol. 19, no. 3, pp. 346-404, 2011.

[29] M. A. Khalighi and M. Uysal, "Survey on free space optical communication: A communication theory perspective," IEEE Commun. Surveys Tuts., vol. 16, no. 4, pp. 2231-2258, Fourthquarter 2014.

[30] Y. Zeng, R. Zhang, and T. J. Lim, "Wireless communications with unmanned aerial vehicles: opportunities and challenges," IEEE Commun. Mag., vol. 54, no. 5, pp. 36-42, May 2016.

[31] Y. Cui, H. Wang, X. Cheng, and B. Chen, "Wireless data center networking," IEEE Commun. Mag., vol. 18, no. 6, pp. 46-53, Dec. 2011.

[32] D. Halperin, S. Kandula, J. Padhye, P. Bahl, and D. Wetherall, "Augmenting data center networks with multi-gigabit wireless links," in $A C M$ SIGCOMM Comput. Commun. Rev., vol. 41, no. 4. ACM, 2011, pp. $38-49$.

[33] S. Mollahasani and E. Onur, "Evaluation of terahertz channel in data centers," in 2016 IEEE/IFIP NOMS, Apr. 2016, pp. 727-730.

[34] C. Jiang, H. Zhang, Y. Ren, Z. Han, K. C. Chen, and L. Hanzo, "Machine learning paradigms for next-generation wireless networks," IEEE Commun. Mag., vol. 24, no. 2, pp. 98-105, Apr. 2017.

[35] G. Alnwaimi, S. Vahid, and K. Moessner, "Dynamic heterogeneous learning games for opportunistic access in lte-based macro/femtocell deployments," IEEE Trans. Commun., vol. 14, no. 4, pp. 2294-2308, Apr. 2015.

[36] X. Cheng, B. Yu, L. Yang, J. Zhang, G. Liu, Y. Wu, and L. Wan, "Communicating in the real world: 3d mimo," IEEE Commun. Mag., vol. 21, no. 4, pp. 136-144, Aug. 2014.

[37] H. Halbauer, S. Saur, J. Koppenborg, and C. Hoek, "3d beamforming: Performance improvement for cellular networks," Bell Labs Tech. J, vol. 18, no. 2, pp. 37-56, Sep. 2013. 